\title{
PROPAGATION OF RADIO WAVES ROUND THE EARTH
}

\author{
By T. L. Eckersley, F.R.S. \\ Marconi's Wireless Telegraph Co.
}

$\mathrm{C}^{\mathrm{s}}$ ONSIDERING the use to which the transmission of radio waves is put, it is remarkable how few have studied the problem. Engineers are interested in the terminal apparatus-the great transmitting and receiving stations; and physicists seem to be more interested in sending radio waves to themserves in the investigation of the ionosphere than in studying the transmission to others across wide open spaces. Both activities are essential to the creation of communication links, but a study of the application of the results obtained by physicists and engineers is also essential.

The mechanism of radio transmission is the same as that of light. Both comprise wave motions in the ether, but the wave-lengths used in radio, although covering a wide range, are very different from those of light.

Radio waves vary from a few centimetres to $20-30$ kilometres, that is, sixteen or more octaves. Light waves are of the order of $10^{-7} \mathrm{~cm}$., and there is only about one octave of visible light. The enormous range of radio waves makes the study of propagation difficult, but at the same time most interesting. Different wave-lengths have been treated pieccmeal by different methods, but there should be, and is, one general method by which all problems can be treated.

The earth is practically a sphere, and for theoretical purposes it can be treated as a homogeneous sphere of uniform electrical conductivity. Irregularities do not matter much to the first approximation.

The propagation of radio waves round such a sphere can be treated mathematically. This was originally done by the great mathematicians such as Poincaré, and others. The mathematics are difficult. They were put on a firm and unimpeachable basis by G. N. Watson at the instigation of Van der Pol. Watson's investigation showed that for long waves the earth behaved as if it were a perfect conductor, and the results are only applicable, without further development, to perfect conductors. The whole theory has now been developed in two independent ways which agree and are applicable whatever the conductivity or inductivity may be:

The results of this mathematical investigation are now clear and definite enough. Waves of which the length is small compared with the radius of the earth are, as in light, practically confined to the region above the plane which is tangent to the earth at the transmitter.
The waves from the transmitter travel out in straight lines, and most, practically all, of the earth is in shadow. Nevertheless, some radio energy can penetrate by diffraction into the shadow region, and the longer the wave-length the more easily it does so.

However this may be, diffraction alone, or bending round the earth, is entirely inadequate to produce a signal at, say, the antipodes on any wave used in radio.

Diffraction is most effective when the wavelength is large, but even the longest waves that can be conveniently used in radio communication are so weak at the antipodes that they need increasing by a factor of the order of $10^{11}$ in order to overcome outside noise, even when the highest powers are used.

It is clear that in the normal radio range it is quite impossible to produce workable signals at the antipodes, or even at distances greater than about 3,000-4,000 kilometres, without the help of some agency which bends the rays round the world. This agency we now know is the ionosphere, which has been largely investigated by physicists in the last ten years. All the long-distance shortwave broadcasts, all the long-distance high-speed beam services, and all the linking up of 99 per cent of the world's telephones would not exist but for the ionosphere. The study of radio transmission round the earth therefore devolves itself into the study of the ionosphere and its effect on longdistance communication.

There are two aspects :

(1) The investigation of the constitution of the ionosphere.

(2) The exploitation of this knowledge in radio communication.

\section{THE IONOSPHERE}

This is a region from 60 to 300 kilometres above the earth, in which a small proportion of the molecules are ionized, so that there is an appreciable atmosphere of electrons capable of reflecting and modifying wireless transmissions.

It is only recently that a detailed and fairly accurate knowledge of it has been obtained, by Appleton in Great Britain and by experiments initiated by Breit and Tuve in the United States.

The method by which the ionosphere, which at 100 or more kilometres is entirely out of direct reach, can be investigated, is very simple in 
essence. Radio impulses a fraction of a millisecond in length are sent up to the ionosphere, where they are reflected down to earth again. From the delay of the reflected echoes, their intensity and polarization, etc., a great many characteristics of the ionosphere can be deduced.

Measurements have been made at a large number of places on the surface of the earth, but the results are by no means sufficient. From these we know that there exists in all places examined a region between 60 and $300 \mathrm{~km}$. above the earth in which there is an appreciable number of free electrons, reaching a concentration as great at $10^{7}-10^{6}$ per c.c. This maximum density is an important characteristic of the layer. It is easily found with the impulse technique already described.

If the radio frequency of the exploring impulses is increased, their delay (that is, the time it takes for the impulses to travel up to the ionosphere and back) rapidly increases, and beyond a certain frequency the rays penetrate the upper layer and echoes cease. This critical frequency gives the maximum electronic concentration in the various layers of which the ionosphere is composed. Near the critical frequency, the curve which is known as the $P^{\prime} f$ curve, relating delay time and radio frequency is split into two nearly circularly polarized branches, this being effected by the earth's magnetic field.

This critical frequency varies with the height of the layer concerned, the time of the day, season, epoch of the sunspot cycle, etc. It is possible, therefore, even with the limited observations at our disposal, to chart the world.

It is found, in effect, that wherever observations are taken, the curves relating delay and frequency are of the same general type, indicating the existence of three main layers, the $E, F_{1}$ and $F_{2}$ layers, approximately at heights of $100 \mathrm{~km}$., $200 \mathrm{~km}$. and $250-300 \mathrm{~km}$.

A great deal has been found out about the ionosphere. Thus the $E$ and $F_{1}$ layers behave normally and vary diurnally, seasonally and with the epoch in the sunspot cycle, as if ionized by the sun's ultra-violet radiation. The $F_{2}$ layer behaves in an anomalous manrier. For example, contrary to expectation, the electron concentration in England is less in summer than in winter. Again, the concentration is different in the northern and southern hemispheres. There appears to be some annual ionizing agency independent of the sun.

By determining the polarization of the two branches of the $P^{\prime} f$ curves near penetration in the northern and southern hemispheres, it has been found that the electron atmosphere is the major factor in guiding wireless waves. A positive ion atmosphere may exist in the lower $E$ layer, but on account of the mass of the positive ions compared with the electrons, these have to be enormously in excess $(10,000$ to 1 or so) in order to affect the wireless waves. All we can say is that, experimentally, this excess does not exist.

One of the major factors in radio transmission is the attenuation of the waves. This is primarily caused by the collision of the electrons with the rest of the molecules. The electrons receive energy from the waves which is wasted as heat in collisions with the molecules.

By making experiments on the loss in reflexion as the impulses are delayed, it is possible to determine the collision frequencies at various heights, this quantity being of the order of $10^{6}$ in the $E$ layer and about $5 \times 10^{3}$ in the $F$ layer.

The ionosphere is disturbed by the mechanism which produces magnetic storms, and also by bright hydrogen eruptions from the sun. This pathology of the ionosphere is of great consequence to communication engineers, and much has been learned about it.

\section{Propagation : Generai}

With the help of this knowledge of the ionosphere, it is possible to get a very fair idea of the propagation of radio waves round the world, and to deduce, for example, which waves are least attenuated and can travel the greatest distances.

Short waves, 10-100 metres, which have been extensively used in world-wide communications, are easiest to understand because ray methods can be used; and, just as in optics, it is possible to trace the rays modified by refraction in the ionosphere. The analysis of these short waves is, in fact, a branch of optics; but when the wavelength becomes comparable with the radius of curvature of the rays, wave methods, and not optics, must be used. Attenuation also largely modifies optical methods, and for exactness and proper understanding, optical methods should be discarded and wave methods used everywhere. Nevertheless, it is sufficient to use optical methods on short-wave ranges.

The correct mathematical method, which should be applicable to the whole of the radio range, is to determine the solution of the equations of propagation in the ionosphere, in the region between the earth and the ionosphere, and in the earth itself, and to satisfy the boundary conditions at the surface of the earth and ionosphere respectively. The difficulty is in obtaining solutions of propagation in the ionosphere, which depend on fourth order differential equations when account is taken of the earth's field.

An approximate development of this is a phase integral method similar to that used in quantum mechanics, which, if the change of phase and 
amplitude on reflexion at the ionosphere are known, is exactly equivalent to the above boundary problems. This phase integral often yields solutions in which methods are much simpler than in the boundary problems, and has been made to give a solution of the diffraction problem in which general relations of great interest are found. These methods-of universal significance-degenerate into optical ray methods for short waves $(10-100$ metres).

\section{SHORT-WAVE TRANSMISSIONS}

The application of our knowledge of the ionosphere is enormously helped by a proposition which translates the vertical $P^{\prime} f$ results into relations for oblique transmission. Thus, if the critical frequency for vertical transmission is known, the critical frequency and consequently skip distance at oblique incidence are also known. This has been checked by an elaborate experiment between Chelmsford and Bodmin. With a sufficiently short wave, even the tangent ray escapes, so that even though it requires a much smaller electron concentration to bend the rays through a small angle, as in long-distance communication, there is ultimately a maximum usable frequency which is about four times the vertical critical frequency. Ground waves can, of course, be used at much higher frequencies. The above theorem is only exactly true if the earth's curvature and steady magnetic field are neglected.

The maximum usable frequency can be shown on a chart in which contours of the quantity are given. To the first approximation, the state of the ionosphere, and hence the contours of this chart, depend only on the sun's position. The chart can be drawn on transparent paper and slid over a map to give the maximum usable frequency for long-distance communication on any route. Complications in transmission may be produced by irregularities in the $E$ layer, which are known to exist, but the cause of which is as yet unknown.

Short-wave transmission is very largely explainable on these optical lines. Each ray usually passes through the $E$ layer up to the $F$ layer, where it is reflected. In the passage through the $E$ layer, it is attenuated by an amount proportional to the square of the wave-length. The shortest waves travel farthest provided they are not too short to be reflected.

\section{LONGER WAVES}

Experiment shows that above about 1,000 metres any increase in the wave-length increases the range, and extreme world-wide ranges can be obtained if waves of the order of $20-30 \mathrm{~km}$. are used. These were originally employed in radio, until it was found that outside noise was so great on these frequencies, and the expense of the gear so high, that the use of such waves became uneconomic.

The behaviour of these waves can easily be explained, but not by ray methods. The boundary value or phase integral wave method must be used.

On these frequencies, $300 \mathrm{kc} . / \mathrm{sec}$. and less, there are no rays in the ionosphere, and we know that all the radio energy is confined to the $E$ layer. The propagation in the $E$ layer is not that of the propagation in a free electron atmosphere. Electron collisions with molecules are predominant, and the electrons do not move freely. The ionosphere behaves like a metallic conductor, and the parameter which controls the type of transmission is the ratio of the collision frequency $v_{c}$ to $\pi$ times the actual frequency $v$. When this quantity $\alpha$ is large, the ionosphere behaves like a conductor; when it is small, like an EcclesLarmor refractive medium. In this, the rays reach a level where the concentration is proportional to the square of the frequency.

In a conductor, on the other hand, there are no rays at all, and the energy only reaches a level which varies inversely as the square root of the frequency.

The transmission may be considered to be guided between two shells, the $E$ region of the ionosphere on the outside and the earth on the inside. The attenuation depends on the resistivity of the shells. This, on account of skin effect, or the diminishing penetration into the layer on these frequencies, increases with increasing frequency; that is, attenuation is proportional to $\sqrt{ } v$, according to the 'Austin Cohen' law.

We therefore obtain the general fact that only extreme wave lengths, the longest and shortest, are useful for long-distance transmission, and this is the characteristic to which the correct treatment by boundary conditions or phase integrals leads. There is a maximum attenuation in day-time on the broadcast band $(500-1,500 \mathrm{kc} . / \mathrm{sec}$.$) and at$ such times the propagation is mostly, if not entirely, carried out by the ground ray, and is independent of the ionosphere. At night-time the layer changes ; $\alpha$ decreases, and there are reflections from the ionosphere and consequent distortions.

The character of transmission depends on the collision frequency in the $E$ layer, and day-time attenuations are a maximum and ranges a minimum on the broadcast band.

Only a general account of radio propagation has been given. Much remains to be done in explaining the vagaries of the ionosphere, but the general characteristics of long-distance radio communication are now well known. 